\title{
BROADBAND SPECTRAL RADIO OBSERVATIONS OF FLARE STARS
}

\author{
A.O. BENZ ${ }^{1}$, M. GÜDEL ${ }^{1}$, T.S. BASTIAN ${ }^{2}$, E. FÜRST ${ }^{3}$, \\ G.M. SIMNETT ${ }^{4}$, L. POINTON ${ }^{5}$ \\ ${ }^{1}$ Institut für Astronomie, ETH-Zentrum, CH-8092 Zürich, Switzerland \\ ${ }^{2}$ National Radio Astronomy Observatory, P.O.Box O, Socorro, NM, USA \\ ${ }^{3}$ Max-Planck-Institut für Radioastronomie, Auf dem Hügel 69, \\ D-5300 Bonn, Federal Republic of Germany \\ ${ }^{4}$ Department of Physics and Space Research, \\ University of Birmingham B 15 2TT, U.K. \\ ${ }^{5}$ The Nuffield Radio Astronomy Laboratories, Macclesfield, \\ Cheshire SK11 9DL, U.K.
}

\section{Summary}

Several nearby flare stars have been observed with spectrometers on various large telescopes. The experience of several attempts to record stellar radio flares is summarized including an event on $\mathrm{AD}$ Leo that was detected by three widely separated large telescopes. The event is analogue to solar decimetric pulsations. An interpretation in terms of coherent cyclotron emission (maser) is given and the source size, exciter drift velocity and source magnetic field are estimated.

\section{Introduction}

The interest in stellar radio burst spectra stems from the solar experience. Different types of solar radio bursts, caused by different emission mechanisms, can easily be distinguished in a time-frequency representation of radio flux density (known as spectrogram). It is therefore an important first step to know the spectra of stellar radio bursts and compare them with those observed on the Sun. This may help to identify the emission mechanism, which allows the determination of coronal parameters of the stellar source.

The first stellar flare radio spectra were taken with the Very Large Array with a bandwidth of $44 \mathrm{MHz}$ and a time resolution of $5 \mathrm{~s}$ by Bastian and Bookbinder (1987). Bastian et al. (1990) have since expanded upon the initial work by using an autocorrelator with the $305 \mathrm{~m}$ telescope at Arecibo. However, further progress on the interpretation of stellar radio bursts can only be achieved by broadband multifrequency observations at high time resolution.

In this paper, the first broadband spectrometer observation of a stellar event is reanalyzed and discussed. The data were originally published in Güdel et al. (1989), but since publication additional timing information has become available which now allows us 
to make a more detailed analysis.

\section{Instruments}

We began these observations with the Jodrell Bank $76 \mathrm{~m}$ telescope using an acousto-optic spectrometer operating over a frequency range of $100 \mathrm{MHz}$. This bandwidth was divided into 128 channels and the spectrum was integrated over $2 \mathrm{~s}$. To discriminate against the terrestrial interference discussed in the next section, we later added the $100 \mathrm{~m}$ telescope in Effelsberg operating at a center frequency of $1665 \mathrm{MHz}$ with total bandwidth of $25 \mathrm{MHz}$ divided into 32 channels and having a time resolution of $0.125 \mathrm{~s}$. For one observing run in November 1987 it was possible to coordinate these observations with the $305 \mathrm{~m}$ telescope in Arecibo operating at $1415 \mathrm{MHz}$ center frequency with a $40 \mathrm{MHz}$ correlator and an effective time resolution of $0.2 \mathrm{~s}$. More details of the instruments are given in Güdel et al. (1989).

Since the appearance of that publication, two unexpected developments allow us to time the common observations of all three telescopes with much higher accuracy than previously reported. First, recent checks of the clock of the Jodrell Bank spectrometer revealed that it runs fast by $8.44 \mathrm{~s} /$ day. Since the clock is manually set for each observation run, an additional timing error of order \pm 1 second may result, which is within the time resolution of the spectrometer. For the work reported here, the first error has been corrected. Second, a check of each scan of the Arecibo data in succession revealed that data gaps of variable duration occured. Once corrected, an absolute timing uncertainty of a few milliseconds was achieved. The timing at Effelsberg was set by the atomic clock of the observatory and is accurate to within a few milliseconds. In practice, we estimate the timing error to be a few tens of milliseconds between Arecibo and Effelsberg.

The timing is now of sufficient accuracy to allow a detailed correlation of the observations in the different frequency bands.

\section{Need for Confirmation of Stellar Origin}

Solar radio astronomers usually can easily recognize terrestrial interference by its structure in the spectrogram. Radio interferometers effectively reject waves from weak terrestrial sources. However, for stellar flare observations with single telescopes the situation is much more difficult and has lead to erroneous results in the past. The problem is that terrestrial interference at the low intensity level of stellar radio flares may resemble the signals we are expecting and can easily be mistaken for them.

An early idea to discriminate terrestrial signals by on-source and off-source measurements turned out to be inefficient if not impossible. We found many similar events on and off source. The type of stellar event shown in the next paragraph occurred only one other time on-source and never off-source. However, the confirmation of its stellar origin by on/off observations would be very time consuming and would always be useful only in a statistical sense. An other idea to discriminate terrestrial signals by off-source monitoring has also proved to be inadequate (see e.g. Bastian et al. 1990). The strategy adopted for the observations reported here serve two purposes: 1) observations with three widely separated telescopes have provided unambiguous confirmation of the stellar origin of the event because there is no source of terrestrial interference that could interfere with all three telescopes simultaneously; 2) the use of three telescopes has allowed us to effectively increase the bandwidth used to observe the event. 


\section{First Broadband Observation}

For the reasons given in the previous paragraph it is more efficient and in fact necessary to observe spectra of stellar flares with widely separated telescopes. After some short and unsuccessful observing sessions we finally recorded a flare on AD Leo with the three telescopes. In the previous report of this event by Güdel et al. (1989) the data of the different telescopes has been shifted in time for the presentation in their figures 1 and 2 . Here we can present the much more accurate timing which basically confirms the previous best fits.

In the case of the Arecibo data, the correction in timing brings the flare observed in Arecibo within $0.4 \mathrm{~s}$ of the best fit with the Effelsberg data (on a different frequency, however). The time correction of the Jodrell Bank observation of the flare brings the observations to within $1.6 \mathrm{~s}$ of the best fit with the Effelsberg data, which is within the time resolution of the Jodrell Bank spectrometer. The corrected timing of the Arecibo and Jodrell Bank observations confirm the common origin of the radiation and eliminate any remaining doubts of the stellar origin of the flare emission.

The full display of data can be seen in the article by Güdel et al. (1989). Figure 1 shows a detailed comparison of the strongest parts of the event. Whereas some peaks seem to correlate, others do not or may be shifted between the Arecibo observation at $1415 \mathrm{MHz}$ and the Effelsberg observation at $1665 \mathrm{MHz}$. We have not found any drifting structures in the single spectrograms of the three telescopes below the instrumental resolution of 200 $\mathrm{MHz} / \mathrm{s}$ in Arecibo and Effelsberg. These lower limits are compatible with the shifts possibly observed in Fig.1. The cross-correlation, presented in Figure 2 for the same time intervals, has a $(>95 \%)$ significant peak at zero lag only in the second $5 \mathrm{~s}$ data segment presented in Figure 1. If all sections are combined, the significance for correlation is less than 90 $\%$. Other peaks at lags different from zero are more significant. This does probably not express a delay of one frequency but quasi-periodicity, as can be seen from the autocorrelation (Fig.3) having a broad peak at $1.1 \mathrm{~s}$. The results indicate that the correlation between the emissions at $1415 \mathrm{MHz}$ and $1665 \mathrm{MHz}$ is weak or absent. Figure 1 also clearly shows that the broadband spectra of the pulses are not similar and most are of smaller bandwidth than the $250 \mathrm{MHz}$ separation.

\section{Discussion}

Having shown that stellar radio bursts can be recorded over broad spectra and may be identified unambiguously as stellar in origin, we now obtain rough estimates of stellar source parameters. From the observed rise times, $\Delta t$, the light dimension of the source of individual pulses, $\Delta s$, may be estimated on the basis of light travel-time arguments: $\Delta s<c \cdot \Delta t$, where $c$ is the speed of light. There have been pulses with rise times faster than the highest time resolution ( $0.1255 \mathrm{~s}$ for the Effelsberg data, cf.Fig.1), thus $\Delta s \lesssim 4 \cdot 10^{9}$ $\mathrm{cm}$.

Another estimate of the source size can be derived from the bandwidth $\Delta \nu$ of an individual pulse. In all coherent solar bursts the emission frequency is related to height by the emission process depending either on the plasma density or, more likely in our case, on the magnetic field. For the latter case, which will be discussed below, we have for the extent of the source in height 


$$
\Delta h \approx \frac{\Delta \nu}{\nu} H_{B}
$$

where $H_{B}$ is the magnetic scale height. Our observations suggest $\Delta \nu \approx 300 \mathrm{MHz}$ at $\nu=1500 \mathrm{MHz}$, thus $\Delta h \approx 0.2 H_{B}$. As an example (and upper limit) we may take a scale height of one stellar radius to estimate $\Delta h \approx 2 \cdot 10^{9} \mathrm{~cm}$.

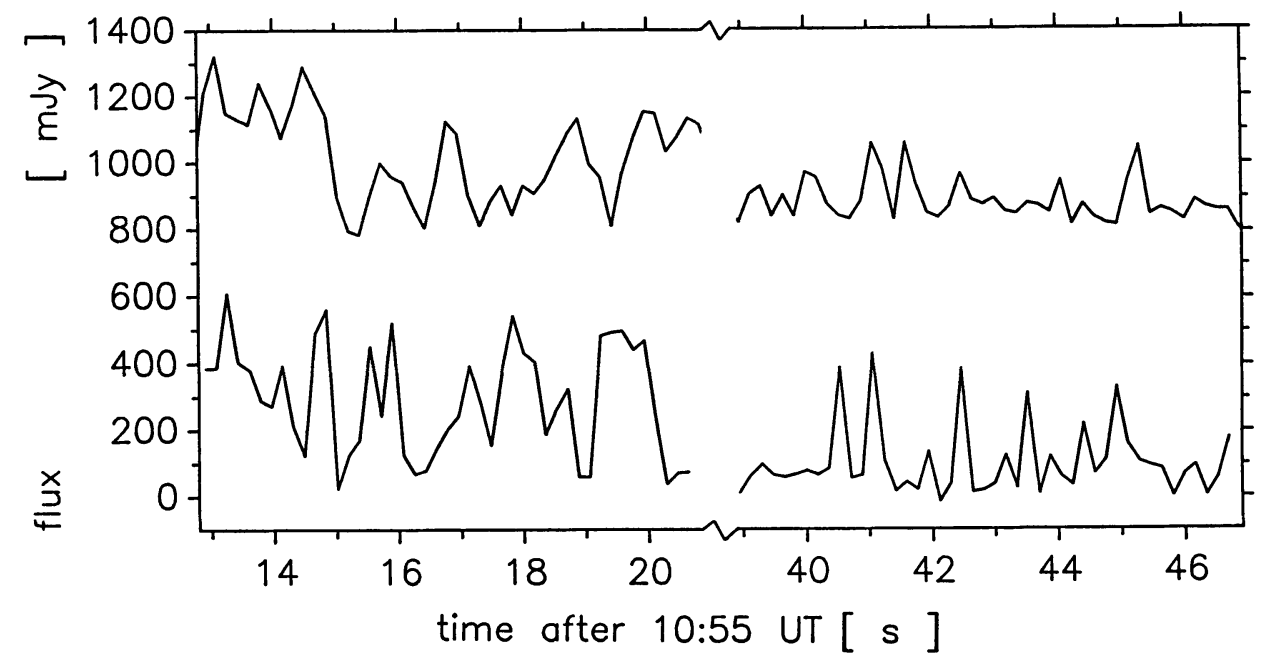

Figure 1: Detailed time profiles of the emission of a radio flare of AD Leo on November 7, 1987 at $1415 \mathrm{MHz}$ integrated over $40 \mathrm{MHz}$ for Arecibo (top) and at $1665 \mathrm{MHz}$ integrated over $25 \mathrm{MHz}$ for Effelsberg (bottom).

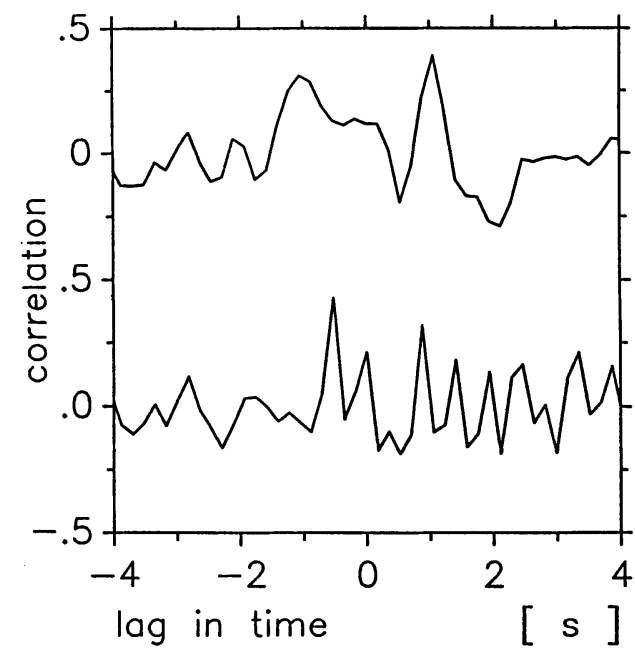

Figure 2: The cross-correlation of the 1415 and $1665 \mathrm{MHz}$ flare emission in the time intervals shown in Fig. 1 (top: first section, bottom: second section).

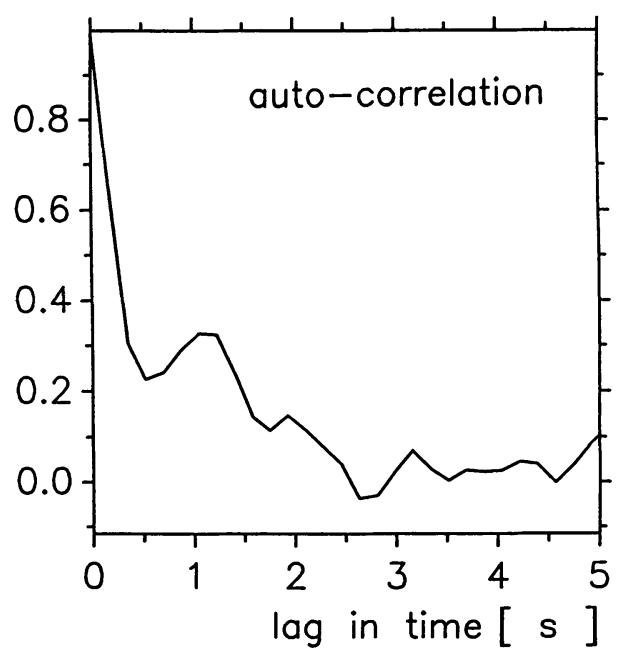

Figure 3: Auto-correlation of the $1415 \mathrm{MHz}$ emission of the radio flare of AD Leo observed at Arecibo. 
The observed flux density $F$ and the estimated source size yield the brightness temperature defined from the Rayleigh-Jeans law

$$
T_{B}=\frac{F \cdot c^{2}}{2 \cdot \nu^{2} \cdot K}\left(\frac{D}{\Delta s}\right)^{2}
$$

where $K$ is Boltzmann's constant and $D$ is the distance to the star. With an observed peak flux of $600 \mathrm{mJy}$ and taking the light dimension as a more reliable estimate we obtain $T_{B}>10^{14} \mathrm{~K}$. This may not be the maximum reported in the literature, but it is the first thoroughly confirmed case.

The AD Leo radio flare is somewhat analogous to solar decimetric pulsations in the $300-1000 \mathrm{MHz}$ band (Güdel and Benz, 1988). Solar decimetric pulsations have bandwidths in the range $\Delta \nu / \nu=0.3-0.5$ and rise times of individual pulses of $0.1-0.5 \mathrm{~s}$. Its quasi-periodic nature (Kurths and Herzel, 1989), high degree of circular polarization (Aschwanden, 1986), and high drift rate (Aschwanden and Benz, 1986) all agree well with the observed stellar parameters. Solar decimetric pulsations are generally attributed to trapped energetic (non-thermal) electrons in magnetic loops having a loss-cone velocity distribution. A model has been developed by Aschwanden and Benz (1988) interpreting the emission by the electron cyclotron instability (generally called 'maser') and the pulsations by relaxation oscillations of the same process.

Adopting the cyclotron maser model suggested by the solar analogy, we can constrain the magnetic field strength in the source if the ratio of plasma frequency to cyclotron frequency is approximately known. The high brightness temperature hints at the most efficient mode of the maser process where this ratio is below unity and the emission is in the fundamental mode. With this assumption a magnetic field strength of $600 \mathrm{G}$ (resp. 300 $\mathrm{G}$, if the emission is at the harmonic) is derived in the source.

\section{Acknowledgements}

The project received partial support by the Swiss National Science Foundation (Grant Nr. 2000-5.499). We are grateful to the staff of the Jodrell Bank Radio Telescope for making the facility available to us for these observations. The Arecibo Observatory is part of the National Astronomy and Ionosphere Center, which is operated by Cornell University under contract with the US National Science Foundation.

\section{References}

Aschwanden, M.J. (1986) Solar Phys., 104, 57.

Aschwanden, M.J. and Benz, A.O. (1986) Astron.Astrophys., 158, 102.

Aschwanden, M.J. and Benz, A.O. (1988) Astrophys.J., 332, 466.

Bastian, T.S. and Bookbinder, J. (1987) Nature, 326, 678.

Bastian, T.S. and Bookbinder, J., Dulk, G.A., and Davis, M. (1989) Astrophys.J., in press (10 April 1990 issue).

Güdel, M. and Benz, A.O. (1988) Astron.Astrophys.Suppl.Ser, 75, 243.

Güdel, M., Benz, A.O., Bastian, T.S., Fürst, E., Simnett, G.M., and Davis, R.J. (1989), Astron.Astrophys., 220, L5.

Kurths, J. and Herzel, H. (1986) Solar Phys., 107, 39. 
LANG: Why are some of the spikes not seen simultaneously at different radio telescopes?

BENZ: The time profiles of the telescopes observing the $\mathrm{AD}$ Leo flare at the same frequency completely agree. The profiles at 1415 and 1665 MHz are not well correlated in < 1 second details. The main reason seems to be that some of the single pulses have a smaller bandwidth. Another reason may be drifts which seem to be both positive and negative. These characteristics are similar to decimetric pulsations if one takes two widely separated frequencies.

LANG: What are the error bars in your radio spectrum of UV Ceti? If they are one sigma you could fit almost any theory to the high frequency data.

BENZ: The possibility to interpret the high frequency part of the quiescent radio spectrum of UV Ceti by another synchrotron emission has been discussed by Gudel and Benz (1989). A more likely interprertation is a low-frequency part of a thermal component. We have shown that a possible candidate is the soft X-ray emitting plasma of UV Ceti using the observed emission measure. The high frequency radio emission may thus correlate with quiescent soft $\mathrm{X}$-ray emission.

LANG: What is the size of the radiating source in your thermal model?

BENZ: It is compatibel with the lower end of the temperature allowed by soft $x$-ray observations and the size of the star, or a higher temperature and a smaller source.

LANG: The quiescent radio emission that has been detected is all about $1 \mathrm{mJy}$, but with two orders of magnitude variation in $\mathrm{x}$-ray luminosity, so it is unlikely the radio and $\mathrm{X}$-ray plasmas are related.

RODONO: I would invite radio astronomers to advise and be advised by other observers at other wavelengths in order to organize simultaneous coverage at several wavelengths. This would also help in selecting genuine flares.

BENZ: The identification of stellar origin of a radio burst by a more or less coincident optical flare has lead to serious misinterpretation in the past. Contrary to interferometers, single dish observations are seriously affected by terrestrial interference. If radio astronomers do not make sure that the observed flare emission is stellar beyond doubt, they cannot start to compare it to optical flares. The association rate between optical and radio flares strongly varies for different solar radio burst types. The situation is unclear for flare stars and needs careful study. We are very interested in collaboration. 